\title{
Nonisothermal Cold Crystallization Kinetics of Poly(ethylene terephthalate)/Clay Nanocomposite
}

\author{
Yaming WANG,${ }^{\dagger}$ Changyu SHEN, and Jinbo CHEN \\ National Engineering Research Center for Advanced Polymer Processing Technology, Zhengzhou University, \\ Zhengzhou 450002, People's Republic of China
}

(Received May 28, 2003; Accepted September 1, 2003)

\begin{abstract}
The nonisothermal cold crystallization behavior of Poly(ethylene terephthalate) (PET) and PET/clay nanocomposite was performed by using differential scanning calorimetry (DSC). Three different kinetics models, namely, the Avrami analysis modified by Jeziorny, the Ozawa model, and a method developed by Mo, were used for the data analysis. The Avrami analysis modified by Jeziorny could gratifyingly describe the primary nonisothermal crystallization stage of PET and PET/clay nanocomposite. The Ozawa analysis failed to provide an adequate description of the nonisothermal cold crystallization of PET and PET/clay nanocomposite. The method developed by Mo was successful in describing the nonisothermal process of PET and PET/clay nanocomposite. It was also concluded that the clay in PET decreased the cold crystallization temperature and the crystallization degree of PET matrix, but did not apparently change the dependence of crystallization peak temperature on heating rate used and the cold crystallization rate of PET matrix.
\end{abstract}

KEY WORDS Poly(ethylene terephthalate) / Clay / Nanocomposites / Crystallization / Differential Scanning Calorimetry /

Poly(ethylene terephthalate) (PET) has been widely used as fibers, bottles, films, and engineering plastics, which hence stimulates the development of PET-based materials. Blending of PET with inorganic particles such as $\mathrm{CaCO}_{3}$, and fiber have been found to enhance crystallization by dense heterogeneous nucleation on the surface of additive particles. ${ }^{1,2}$ However, the obvious phase separation between PET and the inorganic particles is a difficulty, which needs to be solved. Recently, a new class of materials, PET/clay nanocomposites, has been developed by intercalation, followed by in-situ polymerization. ${ }^{3,4}$ Homogeneous dispersion of the nanoscale lamellar clay particles into PET matrix has led to significant improvement in the properties of such nanocomposites, which has unique properties that are not shared by conventional composites, such as high strength, high modulus, high heat distortion temperature, and low gas permeability.

An understanding of dynamic crystallization behavior is practical importance both in the simulation of technological processes and in the determination of parameters of crystallization kinetics of crystallizable polymers. In a preceding study, ${ }^{5}$ we studied the nonisothermal melt crystallization behavior of pristine PET and PET/clay nanocomposites.

The nonisothermal cold crystallization study of PET and PET/clay nanocomposites, which takes place after quenching the materials below the glass transition temperature, followed by heating, is also very impor- tant. From a technical point of view, it is frequently encountered in processing methods such as reheat stretch blow-molding of bottles, heat setting, production of films and fibers, etc. ${ }^{6}$ The physical and mechanical properties of such products are, directly or indirectly, controlled by the crystallization process. From a theoretical point of view, nonisothermal cold crystallization can make contributions to the overall understanding of the crystallization behavior of the polymers.

The aim of the present report is to systematically study the nonisothermal cold crystallization kinetics of pristine PET and PET/clay nanocomposite by a differential scanning calorimetry (DSC). Different theoretical approaches ${ }^{7-10}$ were used to describe the kinetics of nonisothermal crystallization.

\section{EXPERIMENTAL}

\section{Materials and Sample Preparation}

The pristine PET, and PET/clay nanocomposite pellets used in this study were kindly supplied in pellet form by Beijing Yanshan Petrochemical Co., Ltd. (P. R. China). The intrinsic viscosities of the pellets measured in $50 / 50(\mathrm{w} / \mathrm{w})$ of $1,1,2,2$-tetrachloroethane/phenol, at $25^{\circ} \mathrm{C}, 0.1 \mathrm{~g} / 100 \mathrm{~mL}$ concentration, are 0.65 for pristine PET, and 0.82 for PET/clay nanocomposite (with clay weight content of $1.5 \%$ ), respectively. The clay used was montmorrilonite, which was refined and modified by organic intercalated reagent. Sample films approx-

${ }^{\dagger}$ To whom correspondence should be addressed (TEL: +86-371-3887567, FAX: +86-371-3887570, E-mail: wangyaming@zzu.edu.cn). 
imately $0.2 \mathrm{~mm}$ thickness were obtained by hot-press at $280^{\circ} \mathrm{C}$ for several minutes, then quenched by liquid $\mathrm{N}_{2}$.

\section{DSC Measurements}

In this article, a TA 2920 DSC was used to study the crystallization kinetics. Temperature calibration was performed using an indium standard. Sample weight varied between 4.6 and $5.6 \mathrm{mg}$, which was cut from the film already prepared.

The experiment started with heating the sample from room temperature to $280^{\circ} \mathrm{C}$, at selected constant heating rates $\phi$, ranging from 2.5 to $40^{\circ} \mathrm{C} \mathrm{min}^{-1}$. It is noteworthy that each sample was used only once and all the runs were carried out under a nitrogen purge.

\section{RESULTS AND DISCUSSION}

\section{Nonisothermal Cold Crystallization Behavior}

The cold crystallization exotherms of PET and $\mathrm{PET} / \mathrm{clay}$ nanocomposite at five different heating rates ranging from 2.5 to $40^{\circ} \mathrm{C} \mathrm{min}^{-1}$ are presented in Figure 1. Clearly, the exothermic curve becomes wider and shifts to the higher temperature region as the heating rate increases, and these are apparently true for the PET and PET/clay nanocomposite samples studied in this article. The values of the nonisothermal crystallization exotherms peak temperatures $T_{\mathrm{p}}$, and the crystallization enthalpies $\Delta H_{\mathrm{c}}$ of all the samples under different heating rates are collected in Table I. It can be seen that, at a given heating rate, the PET's $T_{\mathrm{p}}>$ PET/clay nanocomposite's $T_{\mathrm{p}}$. This means that the clay in PET decreases the cold crystallization temperature of PET matrix. It can also be seen that, at a given heating rate, the cold crystallization enthalpy $\Delta H_{\mathrm{c}}$ of PET/clay nanocomposite is lower than that of pure PET. Therefore, it can be concluded that the clay in PET decreases the absolute cold crystallization degree of the PET matrix, which is consistent with the results obtained from the melt crystallization of PET/clay nanocomposites. ${ }^{5}$

The relative degree of crystallinity $X(T)$, as a function of crystallization temperature $T$, can be formulated ${ }^{11}$ as

$$
X(T)=\int_{T_{0}}^{T}\left(\frac{d H_{\mathrm{c}}}{d T}\right) d T / \int_{T_{0}}^{T_{\infty}}\left(\frac{d H_{\mathrm{c}}}{d T}\right) d T
$$

where $T_{0}$ and $T_{\infty}$ represent the crystallization onset and end temperature, respectively; $d H_{\mathrm{c}}$, the enthalpy of crystallization released during an infinitesimal temperature range $d T$.

Figure 2 shows the relative degree of crystallinity as a function of temperature for PET and PET/clay nanocomposite at various heating rates. The horizontal temperature scale, such as shown in Figure 2, can be transformed into time domain using the following relationship:

$$
t=\left(T-T_{0}\right) / \phi
$$

where $T$ is the temperature at crystallization time $t$, and $\phi$ is the heating rate. The plots of the relative degree of crystallinity as a function of time for PET and PET/clay nanocomposite at different heating rates are illustrated in Figure 3.

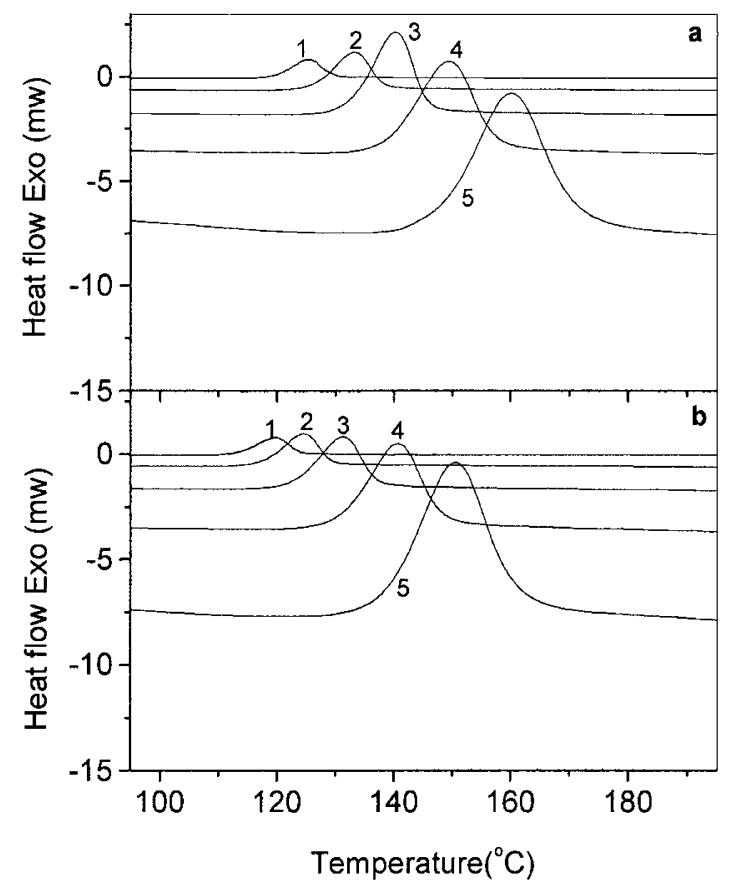

Figure 1. Nonisothermal cold crystallization exotherms of (a) $\mathrm{PET}$, and (b) PET/clay nanocomposite at five different heating rates: $1-2.5^{\circ} \mathrm{C} \mathrm{min}^{-1}, 2-5^{\circ} \mathrm{C} \mathrm{min}^{-1}, 3-10{ }^{\circ} \mathrm{C} \mathrm{min}^{-1}, 4-20{ }^{\circ} \mathrm{C} \mathrm{min}^{-1}$, $5-40{ }^{\circ} \mathrm{C} \mathrm{min}^{-1}$.

Table I. Characteristic data of nonisothermal cold crystallization exotherms for various samples

\begin{tabular}{cccccccc}
\hline \multirow{2}{*}{$\phi\left({ }^{\circ} \mathrm{C} \mathrm{min}^{-1}\right)$} & \multicolumn{3}{c}{ PET } & & \multicolumn{3}{c}{ PET/clay nanocomposite } \\
\cline { 2 - 3 } \cline { 6 - 7 } \cline { 6 - 8 } & $T_{\mathrm{p}}\left({ }^{\circ} \mathrm{C}\right)$ & $t_{1 / 2}(\min )$ & $\Delta H_{\mathrm{c}}\left(\mathrm{J} \mathrm{g}^{-1}\right)$ & & $T_{\mathrm{p}}\left({ }^{\circ} \mathrm{C}\right)$ & $t_{1 / 2}(\min )$ & $\Delta H_{\mathrm{c}}\left(\mathrm{J} \mathrm{g}^{-1}\right)^{\mathrm{a}}$ \\
\hline 2.5 & 125.70 & 6.04 & 29.20 & & 119.99 & 6.15 & 28.42 \\
5 & 133.17 & 3.79 & 35.68 & & 124.71 & 3.15 & 27.07 \\
10 & 140.30 & 2.22 & 37.21 & & 131.21 & 1.76 & 26.05 \\
20 & 149.78 & 1.03 & 30.89 & & 140.78 & 1.09 & 27.04 \\
40 & 160.32 & 0.67 & 34.60 & & 150.59 & 0.64 & 30.88 \\
\hline
\end{tabular}

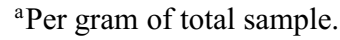




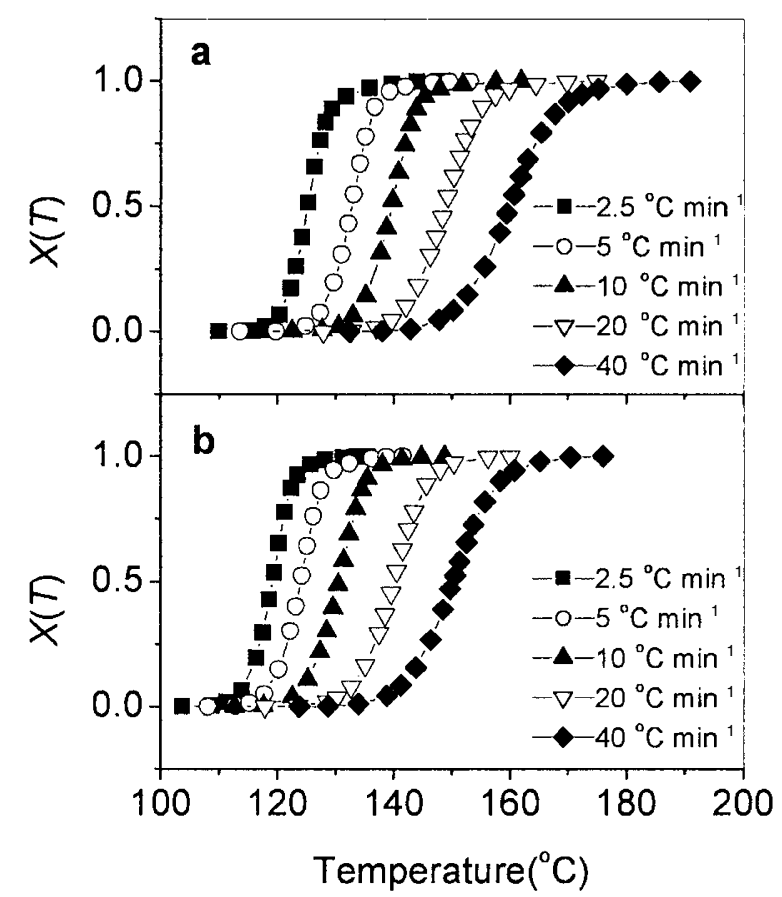

Figure 2. $X(T)$ as a function of temperature for cold crystallization of (a) PET, and (b) PET/clay nanocomposite at five different heating rates.

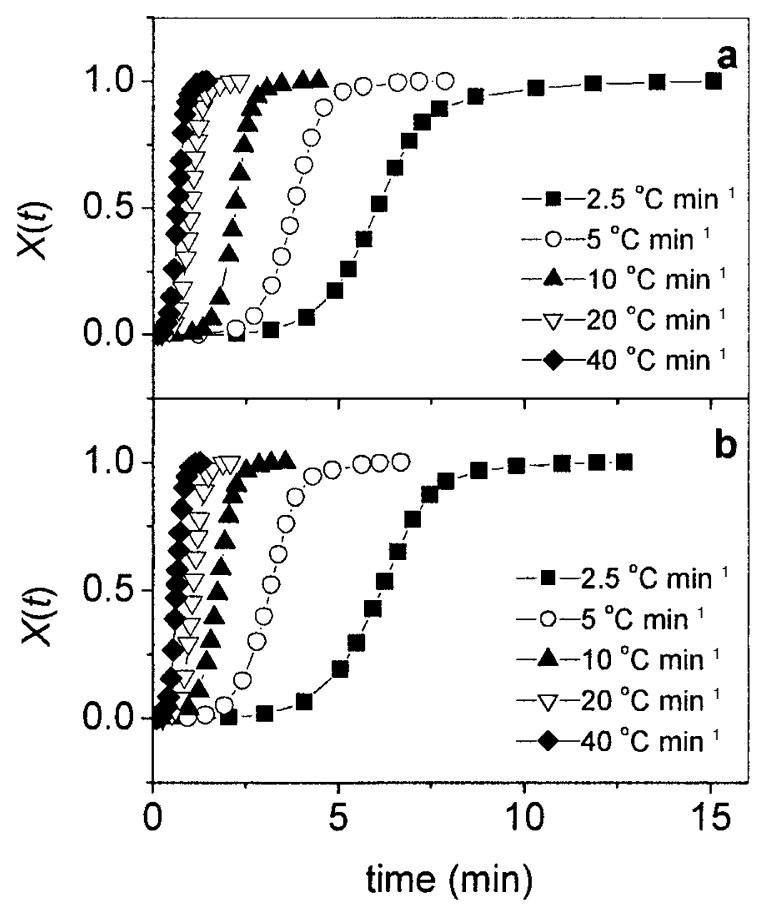

Figure 3. $X(t)$ as a function of time for crystallization of (a) $\mathrm{PET}$, and (b) PET/clay nanocomposite at five different heating rates.

An important parameter which can be taken directly from Figure 3 is the half-time of crystallization $t_{1 / 2}$, which is the change in time from the onset of crystallization to the time at which $X(t)$ is $50 \%$. The $t_{1 / 2}$ of nonisothermal crystallization for PET and PET/clay nanocomposite are listed in Table I. It can be seen that the higher the heating rate, the shorter the time for completing the crystallization. At same heating rate, the PET/clay nanocomposite's $t_{1 / 2}$ is nearly the same as that of PET's. However, at the case of nonisothermal melt crystallization, ${ }^{5}$ the PET/clay nanocomposite's $t_{1 / 2}$ is nearly half of that of PET's. Probably, the reason is that, during nonisothermal melt crystallization, crystallization rate is mainly controlled by nucleation, while, during nonisothermal cold crystallization, crystallization rate is mainly controlled by growth. Therefore, the clay highly accelerates the crystallization rate of PET matrix at the case of nonisothermal melt crystallization but not at the case of nonisothermal cold crystallization.

\section{Nonisothermal Cold Crystallization Kinetics}

The most common approach used to analyze the isothermal crystallization kinetics is the Avrami equation $^{7}$ which assumed that the relative crystallinity $X(t)$ developed with crystallization time $t$,

$$
1-X(t)=\exp \left(-Z t^{n}\right)
$$

where $n$ is the Avrami crystallization exponent, depending on the nature of nucleation and growth geometry of the crystals; $Z$ is the Avrami crystallization rate constant.

Considering the nonisothermal character of the process investigated, Jeziorny ${ }^{8}$ suggested that the value of rate parameter $Z$ should be adequately corrected. The factor that should be considered was the heating rate, $\phi$. Assuming constant or approximately constant $\phi$, the final form of the parameter characterizing the kinetics of nonisothermal crystallization was given as below:

$$
\log Z_{\mathrm{c}}=(\log Z) / \phi
$$

Figure 4 shows the double logarithmic plots of $\log (-\ln [1-X(t)]) v s . \log t$ for PET and PET/clay nanocomposite at various heating rates. Each curve has a linear portion, and which is followed by a gentle deviation at longer times. Usually, this deviation is considered to be due to the secondary crystallization, which is caused by the spherulite impingement in the later stage. Different values for $n$ and $Z_{\mathrm{c}}$ can be obtained from the linear portion of the curves (data at about $5 \%$ to $80 \%$ relative crystallinity are only used for linear regression, and the range is a little difference for each curve), and which are collected in Table II.

For the nonisothermal cold crystallization of PET, $n$ varies from 4.77 to 6.83 . It suggested that the nonisothermal cold crystallization might correspond to three dimensional solid sheaf growth with athermal nucleation. ${ }^{12}$ The same conclusion is reasonable for the the nonisothermal cold crystallization of Poly(aryl ether ether ketone ketone) (PEEKK) ${ }^{10}$ and poly(aryl 


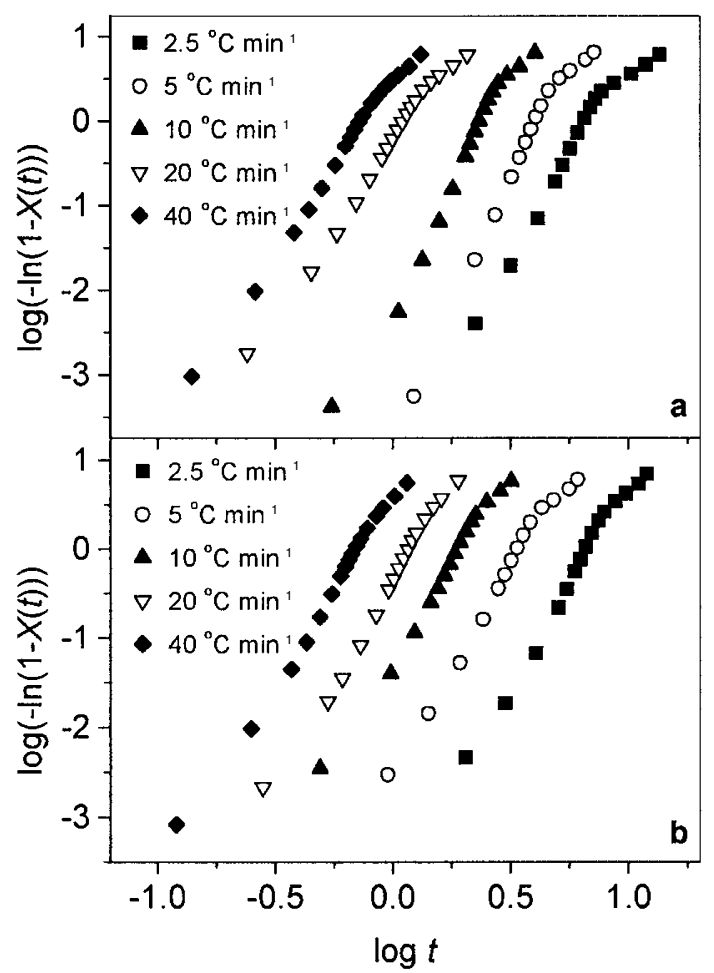

Figure 4. Plots of $\log (-\ln (1-X(t))) v s$. $\log t$ for crystallization of (a) PET, and (b) PET/clay nanocomposite at five different heating rates.

Table II. Nonisothermal cold crystallization kinetic parameters based on Avrami equation modified by Jeziorny

\begin{tabular}{cccccc}
\hline \multirow{2}{*}{$\phi\left({ }^{\circ} \mathrm{C} \mathrm{min}^{-1}\right)$} & \multicolumn{2}{c}{ PET } & & \multicolumn{2}{c}{ PET/clay nanocomposite } \\
\cline { 2 - 3 } \cline { 5 - 5 }$n$ & $n$ & $Z_{\mathrm{c}}$ & & $Z_{\mathrm{c}}$ \\
\hline 2.5 & 5.95 & 0.012 & & 5.05 & 0.013 \\
5 & 6.54 & 0.162 & & 5.25 & 0.272 \\
10 & 6.83 & 0.558 & & 5.23 & 0.717 \\
20 & 4.78 & 0.975 & & 5.35 & 0.960 \\
40 & 4.77 & 1.039 & & 5.74 & 1.097 \\
\hline
\end{tabular}

ether diphenyl ether ketone) (PEDEK).${ }^{13}$ For PET/clay nanocomposite, $n$ varies from 5.05 to 5.74. Obviously, the clay in PET does not apparently affect the way of nucleation and growth of the polymer matrix. The $Z_{c}$ values of PET/clay nanocomposite are nearly the same as that of PET at same heating rate. It can be further concluded that the clay in PET does not apparently affect the cold crystallization rate of the polymer matrix.

Based on the mathematical derivation of Evans, Ozawa ${ }^{9}$ extended the Avrami theory to be able to describe the nonisothermal case. Mathematically, the relative crystallinity can be written as a function of heating rate according to the following equation:

$$
1-X(T)=\exp \left[-K(T) / \phi^{m}\right]
$$

where $K(T)$ is the Ozawa crystallization rate constant; and $m$, the Ozawa exponent (which is similar to the Avrami exponent). Taking the double-logarithmic

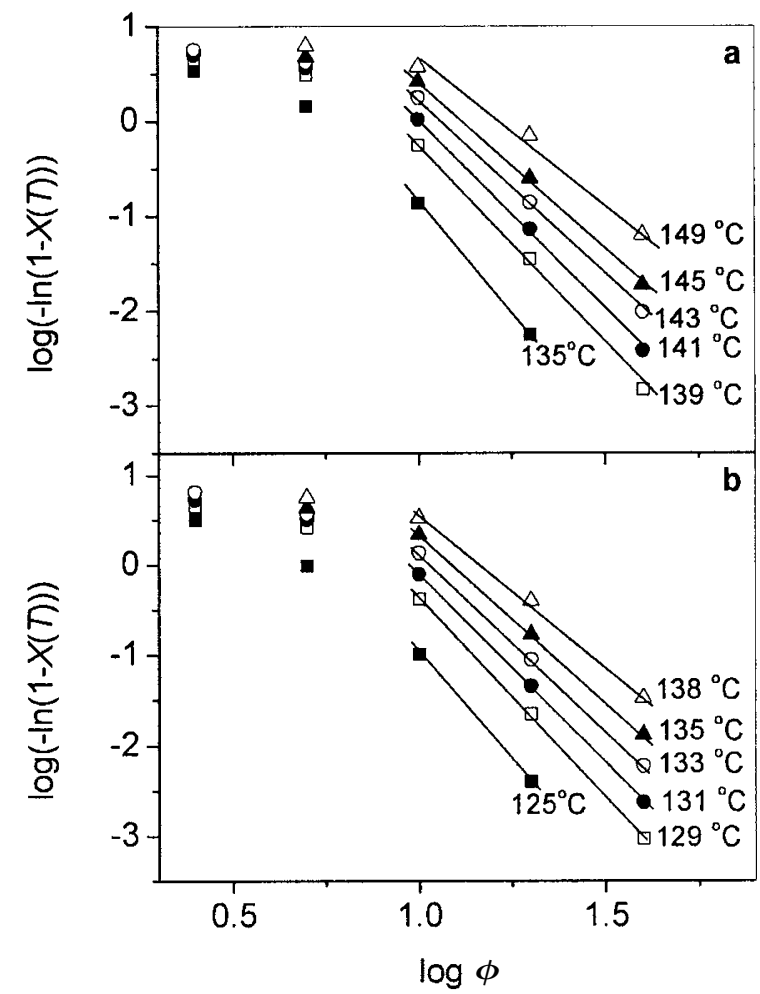

Figure 5. Ozawa Plots of $\log (-\ln (1-X(T))) v s . \log \phi$ for crystallization of (a) PET, and (b) PET/clay nanocomposite.

form,

$$
\log (-\ln (1-X(T)))=\log K(T)-m \log \phi
$$

and plotting $\log (-\ln (1-X(T)))$ against $\log \phi$ at a given temperature, a straight line should be obtained if the Ozawa method is valid. The Ozawa crystallization rate constant $K(T)$ is taken as the antilogarithmic value of the $y$-intercept, and the Ozawa exponent $m$ is taken as the negative value of the slope.

Figure 5 illustrates such plots based on the nonisothermal crystallization data of PET and PET/clay nanocomposite for a series of temperatures. The general curvature seen in Figure 5 makes it impossible to determine the heating function $K(T)$, which is similar to that observed in PEEKK ${ }^{10}$ and PEDEK. ${ }^{13}$ It is important to note that Ozawa equation ignored secondary crystallization. ${ }^{9}$ In fact, from Figure 4 , it can be seen that all the curves have a gentle deviation at longer times. Therefore, the reason that the nonisothermal cold crystallization of PET and PET/clay nanocomposite does not follow the Ozawa equation can be explained as that, at a given temperature, the crystallization processes at different heating rates are at different stages, that is, the lower heating rate process is toward the end of the crystallization process, whereas at the higher heating rate, the crystallization process is at an early stage. By the way, it was reported by Ozawa ${ }^{9}$ that Ozawa model could well describe the nonisothermal melt crystallization kinetics of PET. However, the 
Table III. Nonisothermal cold crystallization kinetic parameters of various samples at different degrees of crystallinities by combination of Avrami-Ozawa equation

\begin{tabular}{crcrr}
\hline$X(t)(\%)$ & 20 & 40 & 60 & 80 \\
\hline \multicolumn{4}{c}{ PET } \\
$F(T)$ & 18.27 & 22.00 & 25.19 & 29.21 \\
$a$ & 1.17 & 1.20 & 1.21 & 1.23 \\
& \multicolumn{4}{c}{ PET/clay nanocomposite } \\
$F(T)$ & 16.77 & 20.41 & 23.41 & 27.15 \\
$a$ & 1.22 & 1.23 & 1.24 & 1.25 \\
\hline
\end{tabular}

cooling rate range used by Ozawa ${ }^{9}$ for the analysis of PET kinetics was from 1 to $4{ }^{\circ} \mathrm{C} \mathrm{min}^{-1}$, which is much narrower than the range from 2.5 to $40^{\circ} \mathrm{C} \mathrm{min}^{-1}$ in this study. In fact, from Figure 4, if we consider the data from 10 to $40^{\circ} \mathrm{C} \mathrm{min}^{-1}$ only, a series of straight line can also be obtained for both PET and PET/clay nanocomposite.

A method developed by $\mathrm{Mo}^{10}$ was also employed to describe the nonisothermal crystallization for comparison. For the nonisothermal crystallization process, physical variables relating to the process are the relative degree of crystallinity $X(t)$, heating rate $\phi$, and crystallization temperature $T$. Both the Ozawa and Avrami equations can relate these variables as follows:

$$
\log Z+n \log t=\log K(T)-m \log \phi
$$

and by rearrangement, its final form is given as below:

$$
\log \phi=\log F(T)-a \log t
$$

where the kinetic parameter $F(T)=[K(T) / Z]^{1 / m}$, refers to the value of heating rate that has to be chosen at the unit crystallization time when the measured system amounts to a certain degree of crystallinity; $a$ is the ratio of the Avrami exponent $n$ to the Ozawa exponent $m$ (i.e., $a=n / m)$. At a given degree of crystallinity, by plotting $\log \phi v s . \log t$ (Figure 6), the values of $a$ and $F(T)$ could be obtained by slopes and intercepts of these lines, respectively (Table III). The value of $F(T)$ systematically increases with raising the relative crystallinity for PET and PET/clay nanocomposite, that means, at unit crystallization time, a higher heating rate should be used to obtain a higher degree of crystallinity. While the values of $a$ are almost constant for each sample, which vary from 1.17 to 1.23 for PET, and from 1.22 to 1.25 for PET/clay nanocomposite. It is clear that this combination method is successful in describing the nonisothermal cold process of $\mathrm{PET}$ and PET/clay nanocomposite, which is also true for PEEKK ${ }^{10}$ and PEDEK. ${ }^{13}$

A method proposed by Kissinger ${ }^{14}$ was frequently used to evaluate the activation energy $\Delta E$ of nonisothermal crystallization. Considering the variation of the

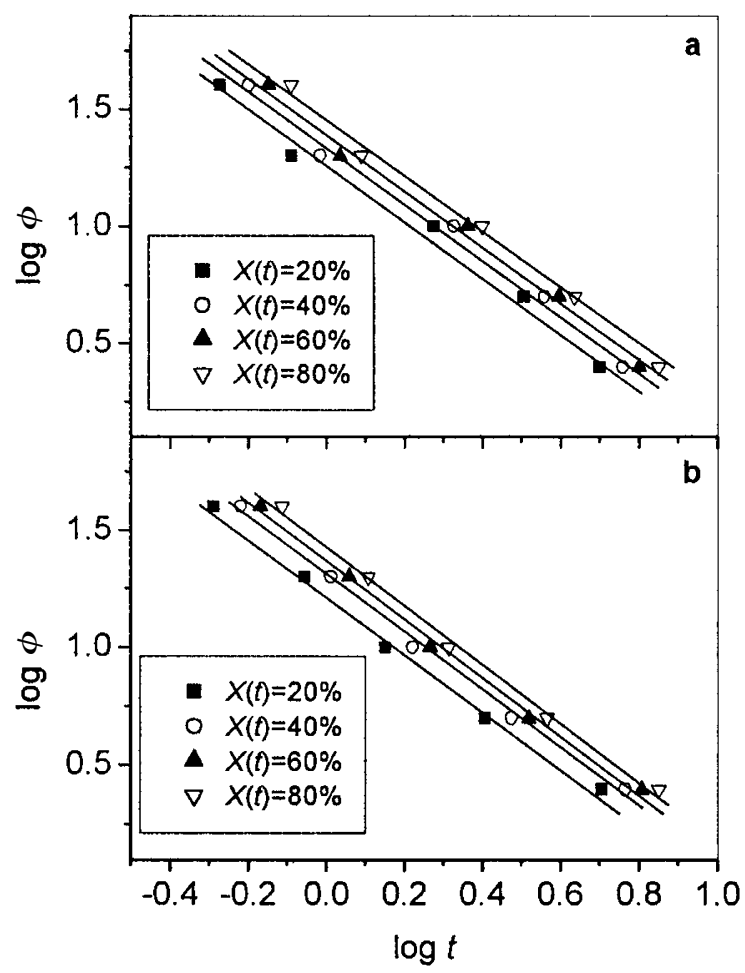

Figure 6. Plots of $\log \phi v s . \log t$ for (a) PET, and (b) PET/clay nanocomposite.

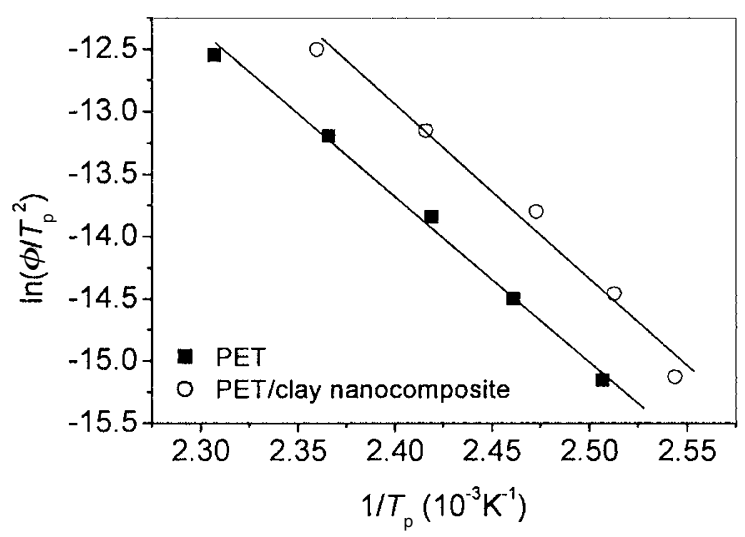

Figure 7. Determination of the activation energy $\Delta E$ describing the nonisothermal crystallization process for PET and PET/clay nanocomposite based on Kissinger method.

peak temperature $T_{\mathrm{p}}$ with the heating rate $\phi$, the effective activation energy $\Delta E$ can be evaluated based on plots of the following forms:

$$
d\left[\ln \left(\phi / T_{\mathrm{p}}^{2}\right)\right] / d\left(1 / T_{\mathrm{p}}\right)=-\Delta E / R
$$

where $R$ is the universal gas constant.

Figure 7 illustrate plots based on the Kissinger method (data from Table I). The slopes of the leastsquare lines drawn through these plots equal $-\Delta E / R$; thus, the effective activation energy $\Delta E$ can be calculated accordingly. The results of $\Delta E$ are $109 \mathrm{~kJ} \mathrm{~mol}^{-1}$ for PET, and $115 \mathrm{~kJ} \mathrm{~mol}^{-1}$ for PET/clay nanocomposite. In our previous work, ${ }^{5}$ a contradictive conclusion was obtained from the results of activation energy $\Delta E$ 
and crystallization kinetics. It suggested that the calculated $\Delta E$ was just an apparent value, which might not truly reflect the activation energy needed by crystallization. However, the above method can at least provide a finite relationship between the peak temperature $T_{\mathrm{p}}$ obtained from the nonisothermal crystallization exotherms and the heating rate used. It can be seen that the calculated $\Delta E$ value for PET/clay nanocomposite is approximately equil to that for PET. Therefore, the clay in PET does not apparently change the dependence of the nonisothermal crystallization exotherm peak temperature $T_{\mathrm{p}}$ on the heating rate $\phi$ of the PET matrix.

\section{CONCLUSIONS}

The nonisothermal cold crystallization data for PET and PET/clay nanocomposite obtained by DSC were analyzed according to three different kinetics models, namely, the Avrami analysis modified by Jeziorny, the Ozawa model, and a method developed by Mo. The Avrami analysis modified by Jeziorny could gratifyingly describe the primary nonisothermal crystallization stage of PET and PET/clay nanocomposite, and the deviation of linearity at the longer time might be ascribed to the occurrence of the spherulite impingement in the secondary stage. The Ozawa analysis failed to provide an adequate description of the nonisothermal crystallization of PET and PET/clay nanocomposite, and this might be due to secondary crystallization. The method developed by Mo was successful in describing the nonisothermal crystallization of pristine PET and $\mathrm{PET} /$ clay nanocomposite. Lastly, the clay in PET decreases the cold crystallization temperature and the crystallization degree of PET matrix, but does not apparently change the dependence of crystallization peak temperature on heating rate used and the cold crystallization rate of PET matrix.

Acknowledgment. The authors gratefully acknowledge the financial support from the Young Standout Teacher Funds of Universities, and the NSF of the Bureau of Education in Henan Province, China (20014300017).

\section{REFERENCES}

1. P. Zhu and D. Ma, Eur. Polym. J., 36, 2471 (2000).

2. V. E. Reinsch and L. Rebenfeld, J. Appl. Polym. Sci., 52, 649 (1994).

3. Y. Ke, C. Long, and Z. Qi, J. Appl. Polym. Sci., 71, 1139 (1999).

4. Y. Ke, Z. Yang, and C. Zhu, J. Appl. Polym. Sci., 85, 2677 (2002).

5. Y. Wang, C. Shen, H. Li, Q. Li, and J. Chen, J. Appl. Polym. Sci., in press.

6. Z. Kiflie, S. Piccarolo, V. Brucato, and F. J. Balta-Calleja, Polymer, 43, 4487 (2002).

7. M. Avrami, J. Chem. Phys., 8, 212 (1940).

8. A. Jeziorny, Polymer, 19, 1142 (1978).

9. T. Ozawa, Polymer, 12, 150 (1971).

10. T. Liu, Z. Mo, S. Wang, and H. Zhang, Polym. Eng. Sci., 37, 568 (1997).

11. P. Cebe and S. D. Hong, Polymer, 27, 1183 (1986).

12. B. Wunderlich, "Macromolecular Physics", Academic Press, Inc., New York, N.Y., 1977, Vol.2.

13. Z. Qiu, H. Zhou, Z. Mo, H. Zhang, and Z. Wu, Polym. J., 32, 287 (2000)

14. H. E. Kissinger, J. Res. Natl. Bur. Stand., 57, 217 (1956). 